\title{
PREVALÊNCIA DE ANEMIA, PERFIL COMPORTAMENTAL E ASPECTOS NUTRICIONAIS EM IDOSOS RESIDENTES DE CIDADE DE PEQUENO PORTE DO SUL DO BRASIL
}

\section{Larissa CASTELACI, Helena ZORASKI, Marilene FIAMETTI, Ramison SANTOS, Maria Luísa de Oliveira GREGOLETTO \& Cleber CREMONESE*}

Centro Universitário da Serra Gaúcha, Caxias do Sul, RS, Brasil.

*Autor para correspondência: clebercre@yahoo.com.br

http://dx.doi.org/10.18571/acbm.113

\section{RESUMO}

A diminuição da capacidade fisiológica e o declínio funcional dos órgãos, associados ao aumento da expectativa de vida, tornam os idosos mais propensos ao desenvolvimento de doenças. A anemia é uma patologia comum nos idosos, associada a várias condições crônicas, com sintomas que podem ser confundidos pela própria idade. Com o objetivo de avaliar a prevalência de anemia e identificar fatores associados em idosos residentes no município de Nova Roma do Sul- RS, foi realizado um estudo transversal. A coleta de dados ocorreu entre março e agosto de 2016, através de visitas domiciliares para aplicação de questionários, que identificaram informações demográficas, comportamentais e alimentares. Foram mensuradas medidas antropométricas e amostras de sangue, para exames bioquímicos. Presença de anemia foi identificada a partir da concentração sanguínea de hemoglobina, seguindo os valores de referência estabelecidos pela OMS ( $<12 \mathrm{~g} / \mathrm{dL}$ mulheres, $<13 \mathrm{~g} / \mathrm{dL}$ homens). Foram avaliados 294 idosos, onde a prevalência geral de anemia foi de $4,4 \%$. Quando associados, os resultados com maior prevalência do desfecho foram: idade avançada (12,5\%), não ter filhos $(11,1 \%)$, dormir 9 horas ou mais $(7,4 \%)$, diagnóstico de diabetes mellitus $(13,0 \%)$ e hipertensão arterial $(7,4 \%)$, e o consumo elevado de alimentos gordurosos $(9,0 \%)$. A regressão logística ajustada foi significativa para diagnóstico de diabetes mellitus e consumo elevado de alimentos gordurosos, apresentando respectivamente odds ratio de $7,97(\mathrm{p}=0,004)$ e 4,01 ( $\mathrm{p}=0,043)$. A prevalência de anemia em idosos residentes de Nova Roma do Sul- RS foi baixa, no entanto, assim como outras doenças que acometem esta população, merece atenção clínica e tratamento adequado.

Palavras-chave: Idosos; Estado Nutricional; Prevalência; Fatores de Risco; Anemia.

\begin{abstract}
The decrease of physiological capacity and the functional decline of organs, associated with the increase of life expectancy, turn elderly people more prone to disease development. Anemia is a common pathology in the elderly, associated with various chronic conditions, with symptoms that may be confounded by age. A cross-sectional study was carried out to evaluate the prevalence of anemia and to identify problems associated with elderly residents in Nova Roma do Sul, RS. Data collection took place between March and August 2016, through home visits for the application of questionnaires, which identified demographic, behavioral and alimentary information. Anthropometric measures and blood samples were also measured for biochemical tests. Presence of anemia was identified from blood hemoglobin concentration, following the WHO established reference values $(<12 \mathrm{~g} / \mathrm{dL}$ women, $<13 \mathrm{~g} / \mathrm{dL}$ men). It was evaluated 294 elderly patients were evaluated, where an overall prevalence of anemia was $4.4 \%$. When associated, the results with the highest prevalence of the outcome were: advanced age $(12.5 \%)$, no children $(11.1 \%)$, sleep 9 hours or more $(7.4 \%)$, history of diabetes mellitus $(13.0 \%)$ and hypertension $(7.4 \%)$, and high consumption of fatty foods $(9.0 \%)$. Adjusted logistic regression was significant
\end{abstract}


for diabetes mellitus and high consumption of fatty foods, presenting respectively an odds ratio of $7.97(\mathrm{p}=0.004)$ and $4.01(\mathrm{p}=0.043)$. The prevalence of anemia in the elderly residents of Nova Roma do Sul - RS was low, however, as well as other diseases that affect this population, it deserves clinical attention and adequate treatment.

Keywords: Elderly; Nutritional Status; Prevalence; Risk Factors; Anemia.

\section{Introdução}

A prevalência de idosos tem aumentado a nível global, tanto em países desenvolvidos, quanto naqueles em desenvolvimento. Em todo o mundo, entre 1970 e 2025, espera-se um aumento de $223 \%$ no número de pessoas idosas (OMS, 2005). Já a nível nacional, em um estudo realizado por Leite et al. (2015) evidenciou-se que entre 2001 e 2011 houve um aumento de 9\% para $12,1 \%$ dessa população. Em termos regionais, no ano de 2015, o Rio Grande do Sul apresentava $15,6 \%$ de sua população total com idade $\geq 60$ anos (FEE, 2015).

Como consequências do envelhecimento, a diminuição da capacidade fisiológica e o declínio funcional dos órgãos tornam os idosos mais propensos ao desenvolvimento de morbidades. Destacam-se, como fator de exposição, associados ao declínio da capacidade funcional desta população, a baixa renda e escolaridade, presença de depressão, de comorbidades, as debilidades cognitivas, a inatividade física e a falta de contato social (MILAGRES et al., 2015a; BUFFON et al., 2015; PERRACINI e FLÓ, 2011).

Neste contexto, verifica-se que o estilo de vida que as pessoas adotam, associado a outros fatores, aumentam a incidência de doenças crônicas. Segundo o Ministério da Saúde (2011), as doenças mais prevalentes são: hipertensão arterial, diabetes mellitus, insuficiência cardíaca, câncer e doenças associadas ao trato respiratório, que correspondem a aproximadamente $70 \%$ das causas de morte no Brasil. Estas doenças podem estar fortemente associadas ao aparecimento de problemas hematológicos, que são comumente encontrados nos idosos (VERAS, 2012; LEITE et al., 2015).

Como consequência, destaca-se a anemia em idosos, que segundo a Organização Mundial da Saúde (WHO,2001), é considerada patológica quando há diminuição da concentração de hemoglobina circulante no sangue, a partir de valores inferiores a $12 \mathrm{~g} / \mathrm{dL}$ para mulheres, e $13 \mathrm{~g} / \mathrm{dL}$ para homens. Muitas vezes os sinais clássicos da anemia (palidez cutâneo-mucosa, dispneia, taquicardia, fadiga, tontura) são confundidos por características inerentes a idade ou pelo uso de alguns medicamentos, apresentando risco elevado de mortalidade (GUALANDRO et al., 2010).

Estudos nacionais apontam prevalência entre 4,5\% e 8,8\% de anemia (BUFFON et al., 2015; SILVA et al., 2012). Essas variações devem-se as diferentes populações estudadas, relacionadas, quase sempre, a menor escolaridade, dificuldade no acesso a saúde e perfil alimentar inadequado. Já, estudos internacionais apontam prevalências maiores de anemia, caso de um recente estudo mexicano, com 1920 idosos, o qual identificou prevalência de anemia em 13,9\% (CONTRERAS-MANZANO et al., 2015), e um estudo peruano, sendo o desfecho presente em 23,3\% dos idosos avaliados (TARQUI-MAMANI et al., 2015).

Ainda, ressalta-se que, são poucos os estudos sobre anemia em municípios de pequeno porte, de forma que o conhecimento da prevalência pode oferecer informações para locais que possuam a mesma característica populacional, e assim direcionar ações de melhoria (MILAGRES et al., 2015b). Desta forma, o presente estudo teve como objetivo determinar a prevalência de anemia e identificar fatores associados em idosos residentes de um município de aproximadamente 3 mil habitantes no sul do Brasil. 


\section{Materiais e Métodos}

\subsection{Delineamento e população de estudo}

Trata-se de um estudo epidemiológico observacional com delineamento transversal, composto por idosos residentes de uma cidade de pequeno porte, na região serrana do Estado do Rio Grande do Sul-RS.

\subsection{Característica amostral}

O cálculo da amostra considerou os seguintes pressupostos: número de idosos residentes na cidade (622 idosos), prevalência de $10 \%$ do desfecho, nível de confiança de $95 \%$, erro amostral de $5 \%$, efeito de delineamento de 1,5 e $20 \%$ para eventuais perdas e recusas.

O sorteio, aleatório e simples, ocorreu através das listas de idosos fornecidas pelas 7 agentes comunitárias responsáveis pela cobertura total do município. Assim, a amostra final foi constituída por 302 idosos, moradores da área urbana e rural do município. Porém, 294 destes participantes apresentaram resultados para o desfecho - presença de anemia - sendo o número utilizado nas análises do presente estudo.

O período de coleta de dados ocorreu entre março a agosto de 2016, incluindo pessoas de idade $\geq 60$ anos, de ambos os sexos, apresentando capacidade cognitiva para responder ao questionário, com condições físicas para a coleta de medidas antropométricas e dispostos a fornecer uma amostra de $7 \mathrm{ml}$ de sangue para realização de exames sanguíneos.

\subsection{Coleta de dados}

Utilizou-se para a coleta de dados, um questionário padronizado e pré-codificado, construído pelos pesquisadores e testado através de um estudo piloto, no mês de fevereiro de 2016, com 20 idosos, escolhidos de forma aleatória no município de Caxias do Sul.

O contato com cada idoso sorteado foi realizado inicialmente pelas agentes comunitárias de saúde do município, as quais foram treinadas antes do início do estudo e a cada 30 dias, afim de garantir a padronização na abordagem e na aplicação dos questionários. Assim, as visitas domiciliares ocorriam em dois momentos: primeiro as agentes comunitárias visitavam o idoso sorteado, explicavam os objetivos, bem como os procedimentos para coleta dos dados da pesquisa, solicitavam a leitura e assinatura do termo de consentimento livre e esclarecido e aplicavam o questionário. Na sequência, dois pesquisadores treinados, visitavam os idosos para coleta das medidas antropométricas e orientavam sobre a coleta de sangue, bem como agendavam o momento para realização da mesma, a qual ocorria no único laboratório do município.

\subsection{Questionário}

O instrumento foi composto por questões sociodemográficas, comportamentais, antropométricas e de hábitos alimentares.

Em relação aos aspectos sociodemográficos (Tabela 1) foram investigadas e categorizadas as seguintes variáveis: sexo (feminino e masculino), idade (coletada de forma contínua e categorizada pelo tercil em 60 a 69 anos, 70 a 79 anos e $\geq 80$ anos), escolaridade (coletada de forma contínua e categorizada em $\leq 3$ anos e $\geq 4$ anos), cor da pele (branca e não branca), estado civil (com companheiro e sem companheiro), renda familiar ( $>2$ salários e $\leq 2$ salários) e quantidade de filhos vivos (coletada de forma contínua e categorizada pelo tercil em $\geq 4$ filhos, 1 a 3 filhos e não possui filhos). 
As variáveis comportamentais e presença de morbidades investigadas (Tabela 2) foram: exercício físico (coletado de forma contínua e categorizada conforme o Questionário Internacional de Atividade Física (IPAQ) em: ativo $\geq 150$ minutos/semana e insuficientemente ativo <150 minutos/semana) (MATSUDO, 2001; BENETI, 2007; GARCIA, 2013), horas de sono (coletada de forma contínua e categorizada através do valor médio em $\leq 9$ horas e $>9$ horas), tabagismo atual (não e sim), etilismo atual (não e sim), diagnóstico de câncer ao longo da vida ou atual (não e sim), diagnóstico de diabetes mellitus (não e sim), diagnóstico de hipertensão arterial sistêmica (HAS) (não e sim) e uso de medicamento para HAS (não e sim).

As variáveis de exames bioquímicos e hematológicos, medidas antropométricas e hábitos alimentares incluíram (Tabela 3): classificação do índice de massa corporal (IMC), determinado através da divisão da massa, em quilogramas, pelo quadrado da altura, em metros (IMC = $\mathrm{kg} /$ altura $\left.^{2}\right)$. Os indivíduos foram classificados como eutrófico $\left(22-27 \mathrm{~kg} / \mathrm{m}^{2}\right)$, baixo peso $\left(<22 \mathrm{~kg} / \mathrm{m}^{2}\right)$ e excesso de peso $\left(>27 \mathrm{~kg} / \mathrm{m}^{2}\right)$ (BRASIL, 2011). Para circunferência abdominal, considera-se risco elevado para doença cardiovascular $>94 \mathrm{~cm}$ para homens e $>80 \mathrm{~cm}$ para mulheres (sem risco e com risco) (VALENTIM, 2012), e glicose alterada, $\geq 100 \mathrm{mg} / \mathrm{dl}$ (normal e alterada) (SBD, 2016). Quanto ao perfil lipídico, foram analisados colesterol total (categorizado em normal $<239 \mathrm{mg} / \mathrm{dl}$ e alterado $\geq 239 \mathrm{mg} / \mathrm{dl}$ ), LDL-colesterol (categorizado em normal $<130 \mathrm{mg} / \mathrm{dl}$ e alterado $\geq 130 \mathrm{mg} / \mathrm{dl}$ ), HDL-colesterol (coletado de forma contínua e categorizado pelo tercil em desejável $>60 \mathrm{mg} / \mathrm{dl}$, aceitável $\geq 40 \mathrm{mg} / \mathrm{dl} \mathrm{a} \leq 60 \mathrm{mg} / \mathrm{dl}$, e baixo $<40 \mathrm{mg} / \mathrm{dl}$ ), e triglicerídeos (normal $<150 \mathrm{mg} / \mathrm{dl}$ e alterado $\geq 150 \mathrm{mg} / \mathrm{dl}$ ) (XAVIER, 2013). Em relação ao perfil hematológico, foram analisados os leucócitos e categorizados em diminuído $\left(<5.000 \mathrm{~mm}^{3}\right)$, normal $\left(5.000-10.000 \mathrm{~mm}^{3}\right)$ e aumentado $\left(>10.000 \mathrm{~mm}^{3}\right)$, e plaquetas em normal $(150.000-400.000 \mu \mathrm{L})$ e diminuído $(<150.000 \mu \mathrm{L})$ (VERRASTRO, et al., 2005).

\subsection{Consumo alimentar}

Os hábitos alimentares foram investigados através de um questionário de frequência alimentar, adaptado de Zanolla et al. (2009), onde para cada alimento, questionou-se quantos dias dos últimos sete dias anteriores à entrevista, o idoso o havia consumido.

Para análise, os alimentos foram agrupados de tal maneira: somadas as frutas, verduras e legumes; somados doces e sobremesas; leite e derivados; alimentos gordurosos e frituras; e o consumo de carnes, e categorizados em $\leq 4 \mathrm{x} /$ semana e $\geq 5 \mathrm{x} / \mathrm{semana}$. Classificou-se o consumo como menos frequente quando o consumo foi entre zero e quatro dias, e para consumo mais frequente quando a ingesta ocorreu em pelo menos cinco dias da última semana (CASTRO et al., 2008).

\subsection{Antropometria}

As medidas antropométricas coletadas para avaliação do estado nutricional incluíram dados da circunferência abdominal, estatura e peso do entrevistado. A circunferência abdominal foi aferida com fita métrica inelástica marca MACROLIFE®, obtida entre o tórax e o quadril, logo acima da cicatriz umbilical, cuja classificação se deu por meio de pontos de corte para circunferência em idosos, onde para homens $>94 \mathrm{~cm}$ indicam risco para doenças cardiovasculares e $>102 \mathrm{~cm}$ para risco aumentado para doenças cardiovasculares, já para mulheres $>80 \mathrm{~cm}$ e $>88 \mathrm{~cm}$ respectivamente (BRASIL, 2011; ABESO, 2009).

A estatura foi obtida com estadiômetro portátil da marca SECA®, modelo 208, com capacidade de $200 \mathrm{~cm}$ e precisão de $0,1 \mathrm{~cm}$ (Seca, Hamburgo, Alemanha), fixado com fita adesiva em uma parede lisa, sem rodapé, utilizando-se procedimento padrão (WHO, 1995). O peso foi obtido com balança digital marca Plenna ${ }^{\circledR}$, com capacidade de $150 \mathrm{~kg}$ e precisão de $100 \mathrm{~g}$ (Plenna 
Especialidades Ltda., São Paulo, Brasil), utilizando-se procedimento padrão (WHO, 1995). As medidas foram feitas em duplicatas e calculada a sua média.

\subsection{Coleta e análise de sangue}

Para identificação do perfil glicêmico e lipídico, os idosos foram encaminhados (em jejum de 12 horas) para o laboratório de análises clínicas da cidade, onde foram dosados: glicemia de jejum pela metodologia glicose oxidase (categorizada em $<100 \mathrm{mg} / \mathrm{dl}$ - normal; $\geq 100 \mathrm{mg} / \mathrm{dl}$ alterado) (SBD, 2016), colesterol total pela metodologia esterase oxidase (categorizado $<239 \mathrm{mg} / \mathrm{dl}$ - normal; e $\geq 239 \mathrm{mg} / \mathrm{dl}$ - elevado), colesterol LDL pela fórmula de Friedewald (categorizado $<130 \mathrm{mg} / \mathrm{dl}$ - normal; e $\geq 130 \mathrm{mg} / \mathrm{dl}$ - elevado) (XAVIER, 2013), e colesterol HDL pela metodologia direto enzimático - colorimétrico (categorizado $>60 \mathrm{mg} / \mathrm{dl}$ - desejável e $<40 \mathrm{mg} / \mathrm{dl}$ baixo) (XAVIER, 2013), e triglicerídeos pela metodologia oxidase (categorizado $<150 \mathrm{mg} / \mathrm{dl}-$ normal; e $\geq 150 \mathrm{mg} / \mathrm{dl}$ - alterado). Já o hemograma foi realizado em contador hematológico automatizado, e os parâmetros normais utilizados nesta pesquisa foram: leucócitos (5.000$10.000 \mathrm{~mm}^{3}$ ), plaquetas $(150.000-400.000 \mu \mathrm{L})$ (VERRASTRO, et al., 2005) e hemoglobina (>12g/dL para mulheres e $>13 \mathrm{~g} / \mathrm{dL}$ para homens) (WHO, 2001).

\subsection{Pressão arterial}

Através do relato de diagnóstico de pressão arterial elevada e/ou quando o idoso fazia uso de medicamentos anti-hipertensivos.

\subsection{Desfecho - Anemia}

Foi identificada pela dosagem de hemoglobina sanguínea, utilizando o ponto de corte proposto pela Organização Mundial da Saúde (WHO, 2001), onde valores inferiores a 12g/dL para mulheres e $13 \mathrm{~g} / \mathrm{dL}$ para homens estão associados a presença de anemia.

\subsection{Análise de dados}

A construção do banco de dados e as análises estatísticas foram realizadas por meio do programa SPSS Statistic Data 20 (Statistical Package for Social Sciences - Chicago, IL, 2008). A análise descritiva das variáveis avaliadas foi realizada por meio de valores absolutos, frequências e prevalências do desfecho. Na sequência foram calculadas as Odds ratio brutas, seus respectivos intervalos de confiança de 95\% (IC95\%) e o p valor. Por fim, na análise multivariada, realizou-se uma regressão logística, levando-se em conta o desfecho dicotômico e a baixa prevalência geral do mesmos $(4,4 \%)$. Utilizou-se a técnica de backwards para análise ajustada, seguindo o critério: as variáveis que atingiram até $20 \%$ de significância $(\mathrm{p} \leq 0,20)$ na análise bruta (tabelas 1,2 e 3 ) foram incluídas no modelo final (tabela 4), no qual foi considerado um nível de significância de $5 \%(\mathrm{p} \leq 0,05)$ para identificar associação entre o desfecho e as variáveis de exposição.

\subsection{Aspectos éticos}

O projeto de pesquisa foi aprovado por um Comitê de Ética em Pesquisa, de acordo com a Resolução ${ }^{\circ}$ 466/12 do Conselho Nacional de Saúde, sob $n^{\circ}$ do parecer 1.402.273. Todos os idosos participantes do estudo leram e assinaram o termo de consentimento livre e esclarecido, o qual autorizou a participação e publicação dos resultados em anonimato. 


\section{Resultados}

A amostra compreendeu 302 idosos, onde 294 apresentaram resultados para os exames de hemoglobina, com prevalência geral de anemia em 4,4\%;

A Tabela 1 apresenta as características sociodemográficas e socioeconômicas da população investigada. Destacam-se, maiores frequências do sexo feminino $(55,8 \%)$, idade de até 69 anos $(51,4 \%)$, baixa escolaridade $(41,8 \%)$ e brancos $(94,6 \%)$. Ainda, idosos que viviam com companheiros, com renda $\leq 2$ salários mínimos e com filhos vivos, representaram, respectivamente, $71,1 \%, 57,1 \%$ e 90,8\%. Em relação ao desfecho, observou-se uma associação significativa em relação ao aumento da idade, onde a anemia estava presente em $12,5 \%$ dos idosos, com 80 ou mais anos, indicando uma chance 7 vezes maior de desenvolver o desfecho nesta faixa etária ( $\mathrm{p}=0,032)$. Em relação a quantidade de filhos, apresentar entre 1 e 3 filhos aumenta a proteção em $85 \%$ quanto a desenvolvimento do desfecho $(\mathrm{p}=0,009)$.

Tabela 1: Descrição das variáveis sociodemográficas e socioeconômicas, prevalência e OR bruta para presença de anemia em idosos residentes em Nova Roma do Sul, RS, 2016. (n=294).

\begin{tabular}{|c|c|c|c|c|}
\hline Variáveis de exposição & n (\%) & Prevalência de anemia $(\%)$ & OR bruta (IC95\%) & p-valor \\
\hline Sexo & & & & 0,200 \\
\hline Feminino & $164(55,8)$ & 3,0 & 1 & \\
\hline Masculino & $130(44,2)$ & 6,2 & $2,09(0,67-6,53)$ & \\
\hline Idade & & & & $\mathbf{0 , 0 3 2}$ \\
\hline 60 a 69 & $151(51,4)$ & 2,0 & 1 & \\
\hline 70 a 79 & $103(35,0)$ & 4,9 & $2,52(0,59-10,77)$ & \\
\hline$\geq 80$ & $40(13,6)$ & 12,5 & $7,05(1,61-30,90)$ & \\
\hline Escolaridade (anos de estudo) & & & & 0,144 \\
\hline$\geq 4$ & $171(58,2)$ & 2,9 & 1 & \\
\hline$\leq 3$ & $123(41,8)$ & 6,5 & $2,31(0,76-7,23)$ & \\
\hline Cor de pele & & & & 0,729 \\
\hline Branca & $278(94,6)$ & 6,2 & 1 & \\
\hline Não branca & $16(5,4)$ & 4,3 & $1,48(0,18-12,13)$ & \\
\hline Estado civil & & & & 0,449 \\
\hline Com companheiro (a) & $209(71,1)$ & 3,8 & 1 & \\
\hline Sem companheiro (a) & $85(28,9)$ & 5,9 & $1,57(0,50-4,94)$ & \\
\hline Renda familiar & & & & 0,742 \\
\hline$>2$ salários & $126(42,9)$ & 4,0 & 1 & \\
\hline$\leq 2$ salários & $168(57,1)$ & 4,8 & $1,21(0,39-3,79)$ & \\
\hline Filhos vivos & & & & 0,009 \\
\hline$\geq 4$ filhos & $106(36,1)$ & 7,5 & 1 & \\
\hline 1 a 3 filhos & $161(54,8)$ & 1,2 & $0,15(0,32-0,74)$ & \\
\hline Não possui filhos & $27(9,2)$ & 11,1 & $1,53(0,38-6,21)$ & \\
\hline
\end{tabular}

Quanto aos aspectos comportamentais (Tabela 2), observou-se que os idosos que dormiam mais de 9 horas possuíam quase 6 vezes mais chances de apresentar anemia $(p=0,009)$. Com relação as morbidades, diagnóstico de diabetes mellitus e hipertensão arterial sistêmica aumenta a chance de desenvolver anemia em 5,8 vezes $(p=0,003)$ e 10,48 vezes $(p=0,002)$ respectivamente. 
Tabela 2: Descrição das variáveis comportamentais e morbidades, prevalência e OR bruta para presença de anemia em idosos residentes em Nova Roma do Sul, RS. 2016. (n=294).

\begin{tabular}{|c|c|c|c|c|}
\hline Variáveis de exposição & n $(\%)$ & Prevalência de anemia & OR bruta (IC95\%) & p-valor \\
\hline $\begin{array}{l}\text { Exercício físico } \\
\text { (minutos/semana) }\end{array}$ & & & & 0,949 \\
\hline Ativos $\geq 150$ & $70(23,8)$ & 4,3 & 1 & \\
\hline Insuficientemente ativos & $224(76,2)$ & 4,5 & $1,04(0,28-3,90)$ & \\
\hline$<150$ & & & & \\
\hline Horas de sono & & & & 0,009 \\
\hline$\leq 9$ horas & $145(49,3)$ & 1,4 & 1 & \\
\hline$>9$ horas & $149(50,7)$ & 7,4 & $5,70(1,24-26,18)$ & \\
\hline Tabagista & & & & - \\
\hline Não & $281(95,6)$ & 4,6 & 1 & \\
\hline Sim & $13(4,4)$ & 0,0 & - & \\
\hline Etilista & & & & 0,269 \\
\hline Não & $185(62,9)$ & 5,4 & 1 & \\
\hline Sim & $109(37,1)$ & 2,8 & $0,50(0,13-1,84)$ & \\
\hline Diagnóstico de câncer & & & & 0,880 \\
\hline Não & $253(86,1)$ & 4,3 & 1 & \\
\hline Sim & $41(13,9)$ & 4,9 & $1,13(0,24-5,28)$ & \\
\hline $\begin{array}{l}\text { Diagnóstico de DM } \\
\text { (glicemia } \geq \mathbf{1 0 0} \text { ) }\end{array}$ & & & & $\mathbf{0 , 0 0 3}$ \\
\hline Não & $240(81,6)$ & 2,5 & 1 & \\
\hline Sim & $54(18,4)$ & 13,0 & $5,81(1,86-18,06)$ & \\
\hline Diagnóstico de HAS & & & & $\mathbf{0 , 0 0 2}$ \\
\hline Não & $132(44,9)$ & 0,8 & 1 & \\
\hline Sim & $162(55,1)$ & 7,4 & $10,48(1,34-81,68)$ & \\
\hline Medicamento para HAS & & & & 0,414 \\
\hline Não & $171(58,2)$ & 2,3 & 1 & \\
\hline Sim & $123(41,8)$ & 7,3 & $3,30(0,99-10,96)$ & \\
\hline
\end{tabular}

Conforme a Tabela 3, idosos com excesso de peso representaram $61,2 \%$ do total da amostra. Em relação a circunferência abdominal, 85\% dos entrevistados possuíam valores que representavam risco para doenças cardiovasculares. Quanto aos exames bioquímicos, alterações nos níveis de glicose foram observados em 39,0\% dos idosos. Quando foram avaliados os hábitos alimentares desses idosos, a anemia teve associação com maior frequência de consumo de alimentos gordurosos, aumentando a chance de desenvolver anemia em 3,45 vezes $(p=0,033)$. 
Tabela 3: Descrição dos exames bioquímicos e hematológicos, medidas antropométricas, hábitos alimentares, prevalência e OR bruta para presença de anemia em idosos residentes em Nova Roma do Sul, RS. 2016. (n = 254 a 294).

\begin{tabular}{|c|c|c|c|c|}
\hline Variáveis de exposição & n $(\%)$ & Prevalência de anemia & $\begin{array}{l}\text { OR bruta } \\
\text { (IC95\%) }\end{array}$ & $\begin{array}{c}\mathbf{p}- \\
\text { valor }\end{array}$ \\
\hline IMC $n=294$ & & & & 0,857 \\
\hline Eutrófico $\left(22-27 \mathrm{~kg} / \mathrm{m}^{2}\right)$ & $94(32,0)$ & 5,3 & 1 & \\
\hline Baixo peso $\left(<22 \mathrm{~kg} / \mathrm{m}^{2}\right)$ & $20(6,8)$ & 5,0 & $0,94(0,10-8,48)$ & \\
\hline Excesso de peso $\left(\geq 27 \mathrm{~kg} / \mathrm{m}^{2}\right)$ & $180(61,2)$ & 3,9 & $0,72(0,22-2,33)$ & \\
\hline Circunferência abdominal $n=294$ & & & & 0,429 \\
\hline Sem risco $(\hat{\delta}<94 \mathrm{~cm} ; \phi+80 \mathrm{~cm})$ & $44(15,0)$ & 6,8 & 1 & \\
\hline Com risco $\left(\partial^{\lambda}>94 \mathrm{~cm} ;\right.$ $\left.>>80 \mathrm{~cm}\right)$ & $250(85,0)$ & 4,0 & $0,57(0,15-2,15)$ & \\
\hline Glicose $n=292$ & & & & 0,594 \\
\hline Normal (<100 mg/dl) & $178(61,0)$ & 3,9 & 1 & \\
\hline Alterada $(\geq 100 \mathrm{mg} / \mathrm{dl})$ & $114(39,0)$ & 5,3 & $1,36(0,44-4,14)$ & \\
\hline Colesterol total $n=291$ & & & & 0,770 \\
\hline Normal $(<239 \mathrm{mg} / \mathrm{dl})$ & $251(86,3)$ & 4,0 & 1 & \\
\hline Alterado $(\geq 239 \mathrm{mg} / \mathrm{dl})$ & $40(13,7)$ & 5,0 & $1,27(0,26-6,01)$ & \\
\hline Colesterol LDL $\mathrm{n}=286$ & & & & 0,298 \\
\hline Normal $(<130 \mathrm{mg} / \mathrm{dl})$ & $202(70,6)$ & 5,0 & 1 & \\
\hline Alterado ( $\geq 130 \mathrm{mg} / \mathrm{dl})$ & $84(29,4)$ & 2,4 & $0,47(0,10-2,18)$ & \\
\hline Colesterol HDL $\mathrm{n}=286$ & & & & 0,710 \\
\hline Desejável (>60mg/dl) & $128(44,8)$ & 4,0 & 1 & \\
\hline Aceitável $(\geq 40 \mathrm{mg} / \mathrm{dl} \mathrm{a} \leq 60 \mathrm{mg} / \mathrm{dl})$ & $145(50,7)$ & 4,8 & $1,25(0,39-4,03)$ & \\
\hline Baixo $(<40 \mathrm{mg} / \mathrm{dl})$ & $13(4,5)$ & 0,0 & - & \\
\hline Triglicerídeos $\mathrm{n}=291$ & & & & - \\
\hline Normal $(<150 \mathrm{mg} / \mathrm{dl})$ & $240(82,5)$ & 5,0 & 1 & \\
\hline Alterado $(\geq 150 \mathrm{mg} / \mathrm{dl})$ & $51(17,5)$ & 0,0 & - & \\
\hline Leucócitos $n=294$ & & & & - \\
\hline Normal (5.000-10.000 $\left.\mathrm{mm}^{3}\right)$ & $269(91,5)$ & 4,8 & 1 & \\
\hline Diminuído $\left(<5.000 \mathrm{~mm}^{3}\right)$ & $21(7,1)$ & 0,0 & - & \\
\hline Aumentado $\left(>10.000 \mathrm{~mm}^{3}\right)$ & $4(1,4)$ & 0,0 & - & \\
\hline Plaquetas $n=254$ & & & & - \\
\hline Normal $(150.000-400.000 \mu \mathrm{L})$ & $232(91,3)$ & 5,2 & 1 & \\
\hline Diminuído $(<150.000 \mu \mathrm{L})$ & $22(8,7)$ & 0,0 & - & \\
\hline $\begin{array}{l}\text { Consumo de frutas, legumes e } \\
\text { verduras } n=294\end{array}$ & & & & - \\
\hline Frequente $(\geq 5 \mathrm{x} /$ semana $)$ & $291(99,0)$ & 4,5 & 1 & \\
\hline Menos frequente $(\leq 4 \mathrm{x} /$ semana $)$ & $3(1,0)$ & 0,0 & - & \\
\hline Consumo de doces $n=294$ & & & & 0,228 \\
\hline Menos frequente $(\leq 4 \mathrm{x} /$ semana $)$ & $113(38,4)$ & 2,7 & 1 & \\
\hline Frequente $(\geq 5 \mathrm{x} /$ semana $)$ & $181(61,6)$ & 5,5 & $2,14(0,57-7,96)$ & \\
\hline \multicolumn{5}{|l|}{$\begin{array}{l}\text { Consumo de leite e derivados } \\
\mathrm{n}=294\end{array}$} \\
\hline Frequente $(\geq 5 \mathrm{x} /$ semana $)$ & $258(87,8)$ & 3,9 & 1 & 0,267 \\
\hline Menos frequente $(\leq 4 \mathrm{x} /$ semana $)$ & $36(12,2)$ & 8,3 & $2,25(0,59-8,61)$ & \\
\hline $\begin{array}{l}\text { Consumo de alimentos gordurosos } \\
\mathrm{n}=294\end{array}$ & & & & $\mathbf{0 , 0 3 3}$ \\
\hline Menos frequente $(\leq 4 \mathrm{x} /$ semana $)$ & $216(73,5)$ & 2,8 & 1 & \\
\hline Frequente $(\geq 5 \mathrm{x} /$ semana $)$ & $78(26,5)$ & 9,0 & $3,45(1,12-10,61)$ & \\
\hline Consumo de carnes $n=294$ & & & & 0,333 \\
\hline Frequente $(\geq 5 \mathrm{x} /$ semana $)$ & $174(59,2)$ & 4,4 & 1 & \\
\hline Menos frequente $(\leq 4 \mathrm{x} /$ semana $)$ & $120(40,8)$ & 5,8 & $1,73(0,57-5,30)$ & \\
\hline
\end{tabular}

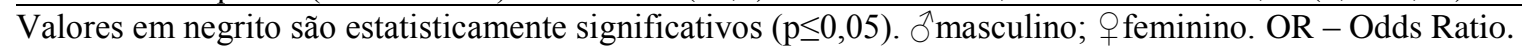

A Tabela 4 apresenta a análise ajustada em relação ao modelo final (variáveis com valor $\mathrm{p} \leq 0,20$ na análise bruta). Assim, mantiveram associação significativa com o desfecho, o relato de diagnóstico de DM e o consumo frequente de alimentos gordurosos, onde indivíduos que referiram diagnóstico de DM apresentaram aproximadamente 8 vezes maior chance de desenvolver anemia 
$(\mathrm{p}=0,004)$ e aqueles idosos com consumo frequente de alimentos gordurosos apresentaram 4 vezes mais chance para desenvolver a morbidade $(\mathrm{p}=0,043)$.

Tabela 4: Regressão logística ajustada em relação a chance de desenvolver anemia em idosos residentes em Nova Roma do Sul, RS. 2016.

\begin{tabular}{|c|c|c|}
\hline Variáveis de exposição & OR ajustada (IC95\%) & p-valor \\
\hline Sexo & & 0,179 \\
\hline Feminino & 1 & \\
\hline Masculino & $2,56(0,65-10,03)$ & \\
\hline Idade & & 0,307 \\
\hline 60 a 69 & 1 & \\
\hline 70 a 79 & $1,49(0,27-8,10)$ & \\
\hline$\geq 80$ & $2,66(0,41-17,32)$ & \\
\hline Escolaridade (anos de estudo) & & 0,673 \\
\hline$\geq 4$ & 1 & \\
\hline$\leq 3$ & $1,36(0,32-5,73)$ & \\
\hline Filhos vivos & & 0,774 \\
\hline$\geq 4$ filhos & 1 & \\
\hline 1 a 3 filhos & $0,27(0,42-1,77)$ & \\
\hline Não possui filhos & $1,30(0,22-7,69)$ & \\
\hline Horas de sono & & 0,195 \\
\hline$\leq 9$ horas & 1 & \\
\hline$\geq 9$ horas & $3,03(0,57-16,16)$ & \\
\hline Diagnóstico de DM & & 0,004 \\
\hline Não & 1 & \\
\hline Sim & $7,97(1,92-33,13)$ & \\
\hline Diagnóstico de HAS & & 0,147 \\
\hline Não & 1 & \\
\hline Sim & $5,12(0,56-46,54)$ & \\
\hline $\begin{array}{l}\text { Consumo de alimentos } \\
\text { gordurosos } n=294\end{array}$ & & $\mathbf{0 , 0 4 3}$ \\
\hline Menos frequente $(\leq 4 \mathrm{x} /$ semana $)$ & 1 & \\
\hline Frequente $(\geq 5 \mathrm{x} /$ semana $)$ & $4,01(1,04-15,42)$ & \\
\hline \multicolumn{3}{|c|}{$\begin{array}{l}\text { Valores em negrito são estatisticamente significativos }(\mathrm{p} \leq 0,05) \text {. } \\
\text { OR - Odds Ratio. Modelo final ajustado pelo método de regressão de } \\
\text { Logística, por todas as variáveis que apresentaram p-valor } \leq 0,20 \text { na análise } \\
\text { bruta. }\end{array}$} \\
\hline
\end{tabular}

\section{Discussão}

O presente estudo encontrou uma prevalência de $4,4 \%$ de anemia nos idosos residentes do município de Nova Roma do Sul-RS. Neste sentido, a prevalência observada foi similar àquela encontrada em estudo de coorte com idosos do município de Bambuí em Minas Gerais (SILVA et al., 2012), onde a maioria dos indivíduos avaliados eram do sexo feminino $(61,9 \%)$ e 4,5\% dos idosos apresentaram o desfecho. Em outro estudo populacional, em Gravataí no Rio Grande do Sul (SCHANN et al., 2007), foram investigados idosos saudáveis em amostra não probabilística, onde a anemia foi diagnosticada em $4,3 \%$ da população. No entanto, a prevalência foi menor, quando comparada com um estudo realizado na Uganda, com idosos acima de 50 anos, onde a prevalência de anemia foi de 20,3\% e esteve associada principalmente a presença de HIV, malária, infecção por helmintos e baixo consumo de frutas (MUGISTA et al., 2013), e também com estudo SADEM (Study on Aging and Dementia in México), realizado no México (JUAREZ-CEDILLO et al., 2014), onde dos 1933 participantes do estudo, 8,3\% eram anêmicos. A baixa prevalência do desfecho pode estar associada, principalmente, ao fato de ser uma cidade de pequeno porte e a 


\section{Biomedica Brasiliensia}

ISSN: 2236-0867

cobertura das agentes de saúde para a população idosa ser praticamente total, facilitando ações de prevenção.

Quando comparadas, a possível associação da anemia com as variáveis de exposição, o estudo apontou associações significativas a um conjunto de variáveis, tais como: idade, filhos vivos, diabetes mellitus, hipertensão arterial e consumo de alimentos gordurosos.

Já é conhecido que a idade elevada traz problemas à saúde, e isso também foi observado com relação ao aumento da prevalência de anemia. Nossos resultados (prevalência de 12,5\% em idosos $\geq 80$ anos) corroboram com outros estudos (BANG et al., 2013; TARQUI-MAMANI et al., 2015; TURUSHEVA et al., 2015) que identificam que a medida que aumenta a idade, aumenta a chance de desenvolver a anemia. $\mathrm{O}$ aumento da prevalência do desfecho em indivíduos mais velhos pode estar associado a diminuição da função hematopoiética da medula óssea, que pode prejudicar as funções corporais e aumentar o risco de morte (YI-CHUN et al., 2016). Sua presença também pode estar relacionada ao aparecimento de doenças e uso de vários medicamentos, dentre eles, os anti-inflamatórios não esteroides, que podem levar ao desenvolvimento de úlcera gástrica, que por sua vez leva a perda sanguínea crônica, podendo resultar em anemia (SGNAOLIN et al., 2013; BUFFON et al., 2015).

Quanto a variável filhos vivos, observou-se que dentre os idosos que não possuíam filhos, $11,1 \%$ apresentaram anemia. Entretanto, indivíduos que possuíam 1 a 3 descendentes, apresentaram um fator de proteção em relação aos que tinham 4 ou mais filhos. Conforme Andrade e Martins (2011), idosos que possuem uma maior funcionalidade familiar apresentam melhor qualidade de vida. Isso pode ser explicado pela diferença entre os cuidados e a atenção dedicados aos idosos pelos familiares próximos, especialmente os filhos, em relação aos cuidadores formais. Ainda, apresentar 4 ou mais filhos pode ser um indicador de pertencer a classes socioeconômicas menos favorecidas.

Os idosos com hábitos de dormir 9 horas ou mais, tiveram maior prevalência de anemia (7,4\%), quando comparados aqueles com menos horas de sono. Um estudo transversal realizado em Campinas, São Paulo, com adultos de 20 anos ou mais, mostra que o padrão de sono de longa duração, está associado ao aumento de morbidades, e também, podem estar relacionados ao uso de medicamentos (FRANCISCO et al., 2014). Neste sentido, alterações no sono podem ser sintomas de doenças ou uso de medicamentos, especialmente para a anemia, visto que um dos principais indicadores desta patologia é a fadiga. Deve-se considerar ainda, que por se tratar de um estudo transversal, não é possível esclarecer a relação causa e efeito desta associação, necessitando, portanto, de estudos para encontrar a relação entre a anemia e maior quantidade de horas de sono.

Já a presença de diabetes mellitus é caracterizada pela hiperglicemia e esteve significativamente associada a anemia, mesmo quando observado o modelo ajustado. Atualmente a diabetes é considerada um dos principais fatores para o desenvolvimento de falha renal, e consequentemente o aparecimento de insuficiência renal crônica. O principal mecanismo para o desenvolvimento de anemia na insuficiência renal crônica é a produção diminuída de eritropoietina e diminuição de disponibilidade de ferro no soro (LOUTRADIS et al., 2016; MILAGRES et al., 2015b; VLAGOPOULOS et al., 2005). Em um estudo realizado em adultos maiores de 18 anos nos Estados Unidos, identificou-se que a anemia foi duas vezes mais prevalente em pessoas com doença renal $(15,4 \%)$ quando comparadas com a população geral $(7,6 \%)$ (STAUFFER e FAN, 2014).

Ainda em relação as morbidades, no modelo bruto a presença de hipertensão arterial sistêmica esteve associada a anemia, corroborando com um estudo de coorte realizado em São Paulo nos anos 2000, 2006 e 2010 (Estudo SABE -saúde, bem-estar e envelhecimento) onde foi verificado que $8,7 \%$ dos idosos que tinham anemia eram hipertensos (CORONA et al., 2014). Em outro estudo transversal realizado por Agarwalla et al. (2016), em uma área de prática de campo na Índia, 41\% dos indivíduos anêmicos tiveram hipertensão, mas este valor não foi significativo 
para a pesquisa. Essa condição está relacionada com a redução da oferta de oxigênio aos tecidos, podendo causar vasodilatação periférica e diminuição da pressão arterial. Para aumentar a eficiência da perfusão, a própria queda da pressão provoca ativação do sistema nervoso simpático e eleva a pressão, levando a taquicardia e vasoconstrição, reduzindo assim, o fluxo sanguíneo renal, o que agrava a condição caso o indivíduo também seja diabético (VASCONCELOS et al., 2013), visto que a hipertensão está associada a um maior grau de resistência à insulina (FRANCISCO et al., 2010).

Quanto a variável consumo de alimentos gordurosos, verificou-se que o consumo mais frequente ( $\geq 5$ vezes por semana) desses alimentos representa uma prevalência maior de anemia, $\mathrm{e}$ aumentou o risco de desenvolver anemia em 4 vezes no modelo ajustado, com IC95\% significativo. Analisando os fatos, estes alimentos possuem grande quantidade de calorias, e o consumo de nutrientes básicos para o organismo, como o ferro, que tem um papel importante no transporte de oxigênio (ANVISA, 2013), muitas vezes são escassos, o que pode desencadear o desfecho aqui tratado. Em um estudo realizado em Minas Gerais, denominado Projeto Bambuí (LOPES et al., 2005), foi realizado um inquérito alimentar para verificar a adequação da ingestão de alimentos da população, onde $36 \%$ dos idosos apresentaram consumo excessivo de gorduras saturadas, e $64 \%$ relataram baixa ingesta proteica, contribuindo assim para o aumento de doenças crônicas não transmissíveis.

O presente estudo não encontrou associação significativa entre anemia e sexo, assim como em outros estudos (BACH et al., 2014; MILAGRES et al., 2015b; CONTRERAS-MANZANO et al., 2015). Conforme Patel (2008) observa-se que a prevalência de anemia é mais baixa em homens quando comparado a mulheres em idade entre 17-49 anos, a medida que, a partir dos 50 anos, esse fato modifica-se, normalmente apresentando valores similares entre os sexos. Isso pode ser explicado porque as perdas menstruais cessam em mulheres idosas. Este fato leva a discussões em relação aos pontos de corte diferentes para determinar a anemia através dos níveis de hemoglobina entre homens e mulheres (BARBOSA et al., 2006; CORONA et al., 2014).

Segundo a Organização Mundial da Saúde (WHO, 2001), a anemia deve ser considerada problema de saúde pública quando a prevalência exceder $5 \%$ da população. No entanto, o resultado encontrado neste estudo é superior ao esperado em uma população saudável (2,5\%). Alguns estudos demostram que a anemia está associada a piores condições de saúde, impactando na mortalidade, principalmente em idosos com outras morbidades (BUFFON et al., 2015; SILVA et al., 2012; GURALNICK et al., 2005). Um hemograma geralmente é suficiente para o diagnóstico dessa condição, no entanto é necessário averiguar a causa com exames complementares, como contagem de reticulócitos, dosagem de ferritina e vitamina $\mathrm{B}_{12}$, nível de anisocitose, creatinina, exames hormonais, entre outros (FAILACE e FERNANDES, 2015)

Os baixos níveis de hemoglobina são acompanhados pela diminuição da massa eritróide com inadequada oxigenação dos tecidos. Algumas complicações mais graves como insuficiência cardíaca, insuficiência circulatória cerebral e potencialização da fragilidade podem ser desencadeadas pela anemia (FAILACE e FERNANDES, 2015)

Dentre as limitações deste estudo, destaca-se o delineamento transversal, onde não é possível determinar a direção das associações, e suas limitações estão relacionadas aos vieses de memória, uma vez que a amostra de idosos pode ter confundido algumas respostas, principalmente no questionário de frequência alimentar. Outra limitação foi à impossibilidade de avaliar as possíveis etiologias da anemia neste grupo estudado.

Como potencial, evidencia-se o percentual da amostra investigada ( $\pm 50 \%$ da população de idosos do município), e a identificação de fatores que contribuem para o surgimento da anemia, tendo em conta, a escassez de estudos sobre o desfecho aqui tratado em municípios de pequeno porte. 


\section{Conclusão}

A prevalência de anemia em idosos residentes em Nova Roma do Sul- RS foi relativamente baixa quando comparada com os resultados observados em outros estudos, no entanto, foi muito similar àquelas encontradas em outras investigações. Desta forma, este estudo evidenciou que os principais fatores desencadeantes para a diminuição dos níveis de hemoglobina são: idade avançada, não ter filhos, dormir 9 horas ou mais, presença de doenças como diabetes mellitus e hipertensão arterial e o consumo elevado de alimentos gordurosos. A anemia no idoso merece atenção clínica e tratamento adequado, da mesma forma que outras doenças que acometem estes indivíduos. Dito isso, devem ser incentivados estudos com indivíduos idosos, visando manter e melhorar as condições de saúde nesta população.

\section{Referências}

ABESO. Associação Brasileira para o Estudo da Obesidade e Síndrome Metabólica. Diretrizes Brasileiras de Obesidade 2009/2010. $3^{\text {a }}$ ed. Itapevi, São Paulo: AC farmacêutica; 2009.

AGARWALLA, R.; SAIKIA, A.M.; PARASHAR, M.; PATHAK, R.; ISLAM, F. Assessment of prevalence of anemia in and its correlates among community-dwelling elderly of Assam, India: A cross-sectional study. Int. J Nutr. Pharmacol Neurol Dis, v. 6, p. 23-27, 2016.

ANDRADE, A.I.N.P.A; MARTINS, R. Funcionalidade familiar e qualidade de vida dos idosos. Millenium, n. 40, p. 185-199, 2011.

ANVISA. Agencia Nacional de Vigilância Sanitária. Anemia por deficiência de ferro. Saúde e economia, ano IV, n. 9, 2013.

BACH, V.; SCHRUCKMAYER, G.; SAM, I.; KEMMLER, G; STAUDER, R. Prevalence and possible causes of anemia in the elderly: a cross-sectional analysis of a large European university hospital cohort. Clin Interv Aging, v. 9, p. 1187-1196, 2014.

BANG, S.M. et al. Anemia and activities of daily living in the Korean urban elderly population: results from the Korean Longitudinal Study on Health and Aging (KLoSHA). Ann Hematol, v. 92, n. 1, p. 59-65, 2013.

BARBOSA, D.L.; ARRUDA, I.K.G.; DINIZ, A.S. Prevalência e caracterização da anemia em idosos do Programa de Saúde da Família. Rev Bras Hematol. Hemoter, v. 28, n. 4, p. 288-292, 2006.

BENEDETTI, T.R.B.; ANTUNES, P.C.; RODRIGUEZ- AÑEZ, C.R.; MAZO, G.Z.; PETROSKI, É.L. Reprodutibilidade e validade do questionário Internacional de Atividade Física (IPAQ) em homens idosos. Rev. Bras. Med. do Esporte; v.13, n.1, p.11-16, 2007.

BRASIL. Ministério da saúde. Secretaria de atenção à saúde. Departamento de atença básica. Orientações para a coleta e análise de dados antropométricos em serviços de saúde: norma técnica do sistema de vigilância alimentar e nutricional- SISVAN. Brasília: Ministério da saúde, 2011. $76 \mathrm{p}$. 


\section{Biomedica Brasiliensia}

ISSN: 2236-0867

BRASIL. Ministério da saúde. Secretaria de Vigilância em Saúde. Departamento de Análise de Situação de Saúde. Plano de ações estratégicas para o enfrentamento das doenças crônicas não transmissíveis (DCNT) no Brasil 2011-2022. Brasília: Ministério da Saúde, 2011. 148 p.

BUFFON, P.L.D.; SGANAOLIN, V.; ENGROFF, P.; VIEGAS, K.; CARLI, G. A. D. Prevalência e caracterização da anemia em idosos atendidos pela estratégia saúde da família. Rev. Bras. Geriatr. Gerontol, v. 18, n. 2, p. 373-384, 2015.

CASTRO, I.R.; CARDOSO, L.O.; ENGSTROM, E.M.; LEVY, R.B.; MONTEIRO, C.A. Surveillance of risk factors for non-communicable diseases among adolescents: the experience in Rio de Janeiro, Brazil. Cad Saúde Pública; v. 24, n. 10, p. 2279- 2288, 2008.

CONTRERAS-MANZANO, A.; DE LA CRUZ, V.; VILLALPANDO, S.; REBOLLAR, R.; SHAMAH-LEVY, T. Anemia and iron deficiency in Mexican elderly population: Results from the Ensanut. Rev Salud Publica de Mex, v. 57, n. 5, p. 394-402, 2015.

CORONA, L.P.; DUARTE, Y.A.O.; LEBRÃO, M.L. Prevalência de anemia e fatores associados em idosos: evidencias do estudo SABE. Rev Saúde Pública, v. 48, n. 5, p. 723-731, 2014.

FAILACE, R.; FERNANDES, F. Hemograma, manual de interpretação (recurso eletrônico), $6^{\circ}$ edição, Artmed, Porto Alegre, 2015.

FRANCISCO, P.M.S.B.; BASTOS, T.F.; COSTA, K.S.; PRADO, M.A.M.B.; BARROS, M.B.A. The use of medication and associated factors among adults living in Campinas, São Paulo, Brazil: differences between men and women. Revista Ciência e Saúde Coletiva, v. 19, n. 12, p. 49094921, 2014.

FRANCISCO, P.M.S.B. et al. Diabetes auto referido em idosos: prevalência, fatores associados e práticas de controle. Cad Saúde Pública, v. 26, n. 1, p. 175-184, 2010.

FUNDAÇÃO DE ECONOMIA E ESTATÍSTICA (FEE). Estimativas populacionais- atualização 2015. Disponível em: <http://www.fee.rs.gov.br/indicadores/populacao/estimativaspopulacionais> Acesso em: 03/10/2016.

GARCIA, M.T.; OSTI, R.F.I.; RIBEIRO, E.H.C.; FLORINDO, A.A. Validação de dois questionários para a avaliação da atividade física em adultos. Revista Brasileira de Atividade Física e Saúde; v. 18, n. 3, p. 317-318, 2013.

GUALANDRO, S. F. M.; HOJAIJ, N. H. S.; FILHO, W. J. Iron deficiency in the elderly. Rev Bras Hematol Hemoter, v. 32, n. 2, p. 57-61, 2010.

GURALNIK, J.M.; ERSHLER, W.B.; SCHRIER, S.L.; PICOZZI, V.J. Anemia in the elderly: a public health crisis in hematology. Hematology Am Soc Hematol Educ Program, v. 1, p. 528532, 2005.

JUÁREZ-CEDILLO, T. et al. Prevalence of anemia and its impact $\mathrm{n}$ the state of frailty in elderly people living in the community: SADEM study. Annals of Hematology, v. 93, n. 12, p. 2057$2062,2014$. 
LEITE M. T., PAI S.D., QUINTANA J. M., COSTA M. C. Doenças crônicas não transmissíveis em idosos: saberes e ações de agentes comunitários de saúde. Revista de Pesquisa Cuidado é Fundamental Online, v. 7, n. 2, p. 2263-2276, 2015.

LOPES, A.C.S.; CAIAFFA, W.T.; SICHIERI, R.; MINGOTI, S.A.; LIMA-COSTA, M.F.L. Consumo de nutrientes em adultos e idosos em um estudo de base populacional: Projeto Bambuí. Cad Saúde Pública, v. 21, n. 4, p. 1201-1209, 2005.

LOUTRADIS, C. et al. Diabetes mellitus increases the prevalence of anemia in patients with chronic kidney disease: a nested case- control study. Word J Nephrol, v. 5, n. 4, p. 358-366, 2016.

MATSUDO, S.M.; ARAÚJO, T.; MATSUDO, V.R. Questionário Internacional de Atividade Física (IPAQ): estudo de validade e reprodutibilidade no Brasil. Rev Bras At Física \& Saúde; v. 6, n. 2, p. 5-18, 2001.

MILAGRES, C.S.; FRANCESCHINI, S.C.C.; PRIORE, S.E.; LIMA, L.M.; RIBEIRO, A.Q. Prevalência e etiologia da anemia em idosos: uma revisão integral. Rev. Medicina, Ribeirão Preto; v. 48, n. 1, p. 99-107, 2015a.

MILAGRES, C. S.; MORAES, K. B. D.; FRANCESCHINI, S. C. C.; SANT'ANA, L. F. R.; LIMA, L. M.; RIBEIRO, A. Q. Prevalência e fatores associados à presença de anemia em idosos do município de Viçosa (MG), Brasil. Ciência \& saúde coletiva, v. 20, n. 12, p. 3733-3741, $2015 b$.

MUGISTA, J.O.; BAISLEY, K.; ASIKI, G.; SEELEY, J.; KUPER, H. Prevalence, types, risk factors and clinical correlates of anemia in older people in a rural Ugandan population. J PLoS ONE, v. 8, n. 10, e78304, 2013.

ORGANIZAÇÃO MUNDIAL DA SAÚDE (OMS). Envelhecimento ativo: uma política de saúde / World Health Organization; tradução Suzana Gontijo. - Brasília: Organização Pan-Americana da Saúde, 2005.

PATEL, K.V. Epidemiology of anemia in older adults. Semin Hematol, v. 45, n. 4, p. 210-217, 2008.

PERRACINI, M.R.; FLÓ C.M. Funcionalidade e envelhecimento. Rio de Janeiro: Editora Guanabara Koogan, 2011.

SCHANN, M.A.; SCHWANKE, C.H.A.; BAUER, M.; LUZ, C.; CRUZ, I.M. Hematological and nutritional parameters in apparently healtly elderly individuals. Rev Bras Hematol Hemoter., v. 29, n. 2, p. 136-143, 2007.

SGNAOLIN, V.; ENGROFF, P.; ELY, L.S.; SCHWANKE, C.H.A.; GOMES, I.; MORRONE, F.B.; DE CARLI, G.A. Hematological parameters and prevalence of anemia among freeliving elderly in south Brazil. Rev Bras Hematol Hemoter, v. 35, n. 2, p. 115-118, 2013.

SILVA, C.L.A.; LIMA-COSTA, M.F.; FIRMO, J.O.A.; PEIXOTO, S.V. Níveis de hemoglobina entre idosos e sua associação com indicadores do estado nutricional e uso de serviços de saúde: Projeto Bambuí. Cad. Saúde Pública, v. 28, n. 11, p. 2085-2094, 2012. 
SOCIEDADE BRASILEIRA DE DIABETES (SBD). Diretrizes SBD 2015-2016. São Paulo: A.C. Farmacêutica, 2016.

STAUFFER, M.E.; FAN, T. Prevalence of anemia in Chronic Kidney Disease in the Unites States. J PLoS ONE, v. 9, n. 1, e84943, 2014.

TARQUI-MAMANI, C. et al. Prevalence of anemia and associated factors in eldery residing in peruvia households. Rev Peru Med Exp Salud Publica, v. 32, n. 4, p. 687-692, 2015.

TURUSHEVA, A. et al. Association between anemia, physical performace, dependency, and mortality in older adults in the north-west region of Russia. J Aging Res Clin Pract, v. 4, p. 3443, 2015.

VALENTIM, A. A. F. Nutrição no envelhecer. São Paulo: Editora Atheneu, 2012.

VASCONCELOS, P.N.; SANTOS, T.M.P.; VASCONCELOS, S.M.L. Consumo de ferro e anemia em mulheres hipertensas e/ou diabéticas. Rev Bras Cardiologia, v. 26, n. 1, p. 17-25, 2013.

VERAS, R.P. Gerenciamento de doença crônica: equívoco para o grupo etário dos idosos. Rev Saúde Pública, v. 46, n. 6, p. 929-934, 2012.

VERRASTRO, T.; LORENZI, T.F.; NETO, S.W. Hematologia e Hemoterapia: Fundamentos de Morfologia, Fisiologia, Patologia e Clínica. São Paulo: Editora Atheneu, $2^{\circ}$ ed., 2005.

VLAGOPOULOS, P.T. et al. Anemia as a risk factor for cardiovascular disease and all-cause mortality in diabetes: the impact of chronic kidney disease. J Am Soc Nephrol, v. 16, p. 34033410, 2005.

WORLD HEALTH ORGANIZATION (WHO). Iron deficiency anemia: assessment, prevention and control: a guide for programed managers. WHO: Geneva; 2001. Disponível em: $<$ http://apps.who.int/iris/bitstream/10665/66914/1/WHO_NHD_01.3.pdf?ua=1 . Acesso em: $01 / 10 / 2016$.

WORLD HEALTH ORGANIZATION (WHO). Physical status: the use and interpretation of anthropometry. Geneva: WHO, $1995 . \quad$ Disponível em: <http://apps.who.int/iris/bitstream/10665/37003/1/WHO_TRS_854.pdf $\geq . \quad$ Acesso em: 07/10/2016.

XAVIER, H.T. et al. Sociedade Brasileira de Cardiologia. V Diretriz Brasileira de Dislipidemias e Prevenção da Aterosclerose. Arq. Bras. Cardiol; v. 101, n. 4, Supl.1, p. 1-22, 2013.

YI-CHUN, H.; JING, C.; MIN, L.; WEIDONG, L.; YANHUA, Y.; LICHEN, Y.; RUI, W.; JIANHUA, P. Study on the anemia status of Chinese urban residents in 2010-2012. Journal of Preventive Medicine, v. 50, n. 3, p. 213-216, 2016.

ZANOLLA, A.F.; OLINTO, M.T.A.; HENN, R.L; WAHRLICH, V.; ANJOS, L.A. Avaliação de reprodutibilidade e validade de um questionário de frequência alimentar em adultos residentes em Porto Alegre, Rio Grande do Sul, Brasil. 